\title{
A Brief Concept of Speech and Language Processing: Parsing, Anaphora, and Fourier Transform
}

\section{Author: Shin-Jye Lee ${ }^{1}$; Ching-Hsun Tseng ${ }^{2}$; Ying-Yi Chou ${ }^{3}$}

\author{
Affiliation: Institute of Management of Technology, National Chiao Tung University, Taiwan ${ }^{13}$; \\ Department of Computer Science, University of Manchester, United Kingdom ${ }^{2}$
}

E-mail: camhero@gmail.com ${ }^{1}$;hank131415go61@ gmail.com ${ }^{2}$; shiaopooh1811@ @mail.com $^{3}$

DOI: 10.26821/IJSHRE.9.6.2021.9615

\begin{abstract}
Due to the quick progressing of computational technology, there are a variety of Natural Language Processing (NLP) technologies applied in this decade, and these technologies surround our daily life in the world. Basically, Speech and Language Processing (SLP) plays an important role in the field of Natural Language Processing, and it promotes an advancéd development to NLP. The primary methods contributing SLP comprise Parsing, Anaphora, and Fourier Transform, and these methods become an increasing issue to carry out a more advanced application of SLP.
\end{abstract}

Keywords: Speech and Language Processing

\section{INTRODUCTION}

Due to the quick progressing of computational technology, there are a variety of Natural Language Processing (NLP) technologies applied in this decade, and these technologies surround our daily life in the world. Basically, Speech and Language Processing (SLP) plays an important role in the field of Natural Language Processing, and it promotes an advanced development to NLP. The primary methods contributing SLP include Parsing, Anaphora, and
Fourier Transform, and these methods become an increasing issue to carry out a more advanced application of SLP. Parsing, Syntax Analysis, the process of deciding whether a string of input symbols is a sentence of a given language and if so determining the syntactic structure of the string as defined by a grammar (it's usually context-free grammar) for the language. In addition, Reference to an entity that has been previously introduced into the discourse is called anaphora. Moreover, Fourier Transform is amathematical operation that analyzes an arbitrary waveform into its constituent sinusoids.

\section{PARSING}

Parsing, Syntax Analysis, the process of deciding whether a string of input symbols is a sentence of a given language and if so determining the syntactic structure of the string as defined by a grammar (it's usually context-free grammar) for the language. This is achieved by means of a program known as a parser or syntax analyzer. For example, a syntax analyzer of arithmetic expressions should report an error in the string 1-+2, since the juxtaposition of the minus and plus operators is invalid. On the other hand the string 1-2-3 is a valid arithmetic expression with structure specified by the statement that its subexpressions are $1,2,3$ and 1-2. The input to a parser is a string of tokens 
supplied by a lexical analyzer. Its output may be in the form of a parse tree or a derivation sequence.

Briefly, Parsing means taking an input and producing some sort of structure for it. Also, Parsing can be viewed as a search problem. Two common architectural metaphors for this research are top-down, which starts with the root $\mathrm{S}$ and grows trees down to the input words. And bottom-up, which starts with the words and grows trees up toward the root S. Moreover, Top down parsers can be made more efficient by using a left-corner table to only suggest non-terminals which are compatible with the input.

\section{ANAPHORA}

Reference - in NLP, is the problem/methods of deciding to what real-world objects various natural language expressions refer. Reference is the process by which speakers use expressions like John to denote a person named John. Secondly, Anaphor - an expression that refers back to a previous expression in a natural language discourse. For example: "John fell sick of a serious illness. He was very weak." The word he refers to John, and is described as an anaphoric reference to John. Thirdly, Anaphora - Reference to an entity that has been previously introduced into the discourse is called anaphora.

\section{FOURIER TRANSFORM}

A mathematical operation that analyzes an arbitrary waveform into its constituent sinusoids. This relationship is stated as

$\mathrm{S}(f)=\int_{-\infty}^{\infty} s(t) \exp (-2 \pi i f t) d t$

where $\mathrm{s}(\mathrm{t})$ is the waveform to be decomposed into a sum of sinusoids, $\mathrm{S}(\mathrm{f})$ is the Fourier Transform of $\mathrm{s}(\mathrm{t})$, and $i=\sqrt{ }-1$. An analogous formula gives $s(t)$ in terms of $\mathrm{S}(\mathrm{f})$, but with a normalizing factor, $1 / 2 \pi$. Sometimes, for symmetry, the normalizing factor is split between the two relations.
The Fourier Transform pair, $s(t)$ and $S(f)$, has to be modified before it is amenable to computation on a digital computer. This modified pair, called the Discrete Fourier Transform (DFT) must approximate as closely as possible the continuous Fourier Transform. The continuous time function is approximated by $\mathrm{N}$ samples at time intervals $\mathrm{T}: \mathrm{g}(\mathrm{kT})$, $\mathrm{k}=0,1, \ldots \mathrm{n}-1$. The continuous Fourier Transform is also approximated by $\mathrm{N}$ samples at frequency intervals 1/NT: $G(n / N T), n=0,1, \ldots N-1$. Since the $N$ values of time and frequency are related by the continuous Fourier Transform, then a discrete relationship can be derived: $\mathrm{G}(\mathrm{n} / \mathrm{NT})=\sum_{k=0}^{N-1} g(k t) \exp (-2 \pi i n k / N)$

\section{CONCLUSION}

Speech and Language Processingis one of popular fields of Natural Language Processing with a splendid future, and it playsa critical role in Natural Language Processing. Although it has been highly developed around the world, there are still a lot of fields that need probing and exploring.

\section{REFERENCE}

[1] Daniel Jurafsky and James Martin, Speech and Language Processing, Prentice Hall, 2000.

[2] Xuedong Huang, Alex Acero and Hsiao-Wuen Hon, Spoken Language Processing, Prentice Hall, 2001.

[3] Christopher Manning and Hinrich Schutze, Foundations of Statistical Natural Language Processing, MIT Press, 1999.

[4] http://www.cse.unsw.edu.au/ billw/nlpdict.html 